\title{
Entangling atoms in photonic crystals
}

\author{
Martin Konôpka ${ }^{1}$ and Vladimír Bužek ${ }^{1,2}$ \\ ${ }^{1}$ Faculty of Mathematics and Physics, Comenius University, Mlynská dolina, 842 15 Bratislava, Slovakia \\ 2 Institute of Physics, Slovak Academy of Sciences, Dúbravská cesta 9, 84228 Bratislava, Slovakia
}

(18 January, 1999)

\begin{abstract}
We propose a method for entangling a system of two-level atoms in photonic crystals. The atoms are assumed to move in void regions of a photonic crystal. The interaction between the atoms is mediated either via a defect mode or via resonant dipole-dipole interaction. We show that these interactions can produce pure entangled atomic states. We analyze the problem with parameters typical for currently existing photonic crystals and Rydberg atoms. We show that the atoms can emerge from photonic crystals in entangled states. Depending on the linear dimensions of the crystal and on their velocity of the entangled atoms can be separated by tens of centimeters.
\end{abstract}

\section{INTRODUCTION}

Quantum entanglement is one of the most remarkable feature of quantum mechanics. Coherent control of the entanglement between quantum systems attracts lot of attention mainly because of its potential application in quantum information processing. Simultaneously, experimental investigation of the entanglement allows us to test basic postulates of quantum mechanics and to answer fundamental epistemological questions. These questions are related to the original Gedanken experiment of Einstein, Podolsky and Rosen [1] which triggered discussions about non-locality of quantum mechanics and motivated experimental proposals to test whether quantum mechanics is the complete non-local theory. The first experimental confirmation of the violation of Bell's inequalities [2] has been done with the help of entangled photons [3]. A weak point of experiments with photons is an insufficient control of directions of emitted photons and small detectors efficiencies. This problem should be removed in proposals where highly excited (Rydberg) atoms are entangled. Probably the first proposal of such an experiment is described in Ref. [1]. Other proposal have been presented in Refs. [5]. Authors of these schemes proposed techniques how to create entangled atoms in microwave single-mode cavities. Recently, controlled entanglement between atoms separated apr. by $10 \mathrm{~mm}$ interacting with an electromagnetic field in a high- $Q$ cavity has been experimentally realized [6]. In addition, trapped ions have been created in entangled states [7].

In this paper we propose a simple scheme for entangling atoms in photonic crystals. We remind us that photonic crystals are artificially created three-dimensional periodic dielectric materials which exhibit a frequency gap or several gaps in spectrum of propagating electromagnetic (EM) waves [8]9]. An EM wave with its frequency from the gap can not propagate in the structure in any direction. Photonic crystals operating at microwave frequencies were successfully created in laboratories [10]. They consist of a solid dielectric and empty regions. The periodicity of a photonic crystal can be destroyed by removing or adding a piece of material which creates a defect EM mode in the structure. This mode is spatially localized around the region of the defect. The frequency of the mode and the spatial modulation of its electric field amplitude depends on properties of the defect 11 14. It means that one can adjust parameters of the defect mode by creating a suitable defect in the crystal. In particular, the spatial dependence of the mode amplitude can be adjusted to particular needs. In quantum optics, defect modes in photonic crystals can be used similarly as high- $Q$ single-mode cavities [15, 16]. The quality factor of a single mode in a metallic cavity can be of order of $10^{8}$ or more and similar values can be reached for a single defect mode in a photonic crystal [15]. Today threedimensional photonic crystals are available only at microwave frequencies. They can be used for experiments with Rydberg atoms, similarly as microwave cavities.

In this paper we consider two interactions via which one can produce entangled atoms. Firstly, we show that at it is possible to generate entangled atoms without a defect mode, using the action of the resonant dipole-dipole interaction (RDDI) [17,18] mediated by offresonant modes of photonic band continua. Secondly, we explore the scheme in which the atoms become mutually entangled due to the interaction with the defect-field mode.

The paper is organized as follows: Basic features of the proposed setup are described in Section III. In Section III we discuss how the atoms in photonic crystals can be entangled via the resonant dipole-dipole interaction. In Section IV we study in detail the entanglement of atoms which interact with a single defect mode in the photonic crystal. In Section $\square$ we conclude the paper with some remarks.

\section{SETUP OF THE SCHEME}

We consider two mechanisms via which a system of identical atoms can be entangled in photonic crystals. We assume that the atoms are modeled by two-level systems having their transition frequencies in a photonic bandgap (PBG). 
The first mechanism is the resonant dipole-dipole interaction (RDDI) mediated by off-resonant modes of the photonic-band continua [see the Hamiltonian (8)]. This interaction has been analyzed in detail by Kurizki 19 and John and Wang [17] as well as by John and Tran Quang [18]. These authors have considered a system of two-level atoms. They have shown that if one of the atoms is excited and the other one is in its ground state, then they can exchange excitation in spite of the fact that their transitions frequencies are in a PBG and spontaneous emission is nearly totally suppressed. The RDDI can be understood as an energy exchange via localized field [17]. This light tunneling (or photon-hopping conduction) can be very efficient when the distance between the atoms is much smaller than the light wavelength. The RDDI can occur either in a free space or in a cavity. However, in a free space the excitation is irreversibly radiated into the continuum of the field modes after a very short time (given by Fermi's Golden rule) and the entanglement between the atoms is deteriorated rapidly.

The second mechanism is due to an excitation exchange via a defect mode which is resonant (or nearly resonant) with the atoms. This type of interaction explicitely involves a quantized defect mode and is described by the Hamiltonian (10).

These two interactions can also occur simultaneously. As we will see, the second mechanism is much more efficient and allows a coherent control over the process of entanglement. The first mechanism can be neglected in many cases, especially when the atoms have their transition frequencies near the center of a wide PBG and their distance is not much smaller than the wavelength of the resonant light.

In what follows we describe the basic setup of the proposed experiment in the case when the atoms interact only via the defect mode. Let us assume that one of the three atoms (let say the atom $A$ ) is prepared initially in its excited state while the other two atoms $(B$ and $C$ ) are initially in their ground states (see Fig.11). After the preparation the atoms are injected into cylindrical void regions of the crystal. We consider the photonic crystal of the geometry designed by Yablonovitch et al. [10,15] although other appropriate geometries can be used as well. The void cylinders intersect at the center of the crystal. The defect-field mode (located near the center of the crystal) is initially prepared in its vacuum state. Firstly the atoms propagate freely in the void cylinders outside the defect-field (this is due to the fact that the transition frequencies of the atoms lie inside the wide PBG). When the atoms enter the defect region they start to interact with the single defect-field mode. And then again, after they leave the defect region they evolve freely. If the exited (ground) state of atom $j(j=A, B, C)$ is denoted as $\left|e_{j}\right\rangle\left(\left|g_{j}\right\rangle\right)$ and the $n$-photon state of the single mode defect field is denoted as $|n\rangle$ then the initial state of the system under consideration can written as

$$
|\Psi(0)\rangle=\left|e_{A}\right\rangle \otimes\left|g_{B}\right\rangle \otimes\left|g_{C}\right\rangle \otimes|0\rangle \equiv\left|e_{A}, g_{B}, g_{C}, 0\right\rangle .
$$

When we assume that in the defect region the atom-field interaction is governed by the Hamiltonian in the dipole and rotating wave approximations (see below) then the final state of the system reads

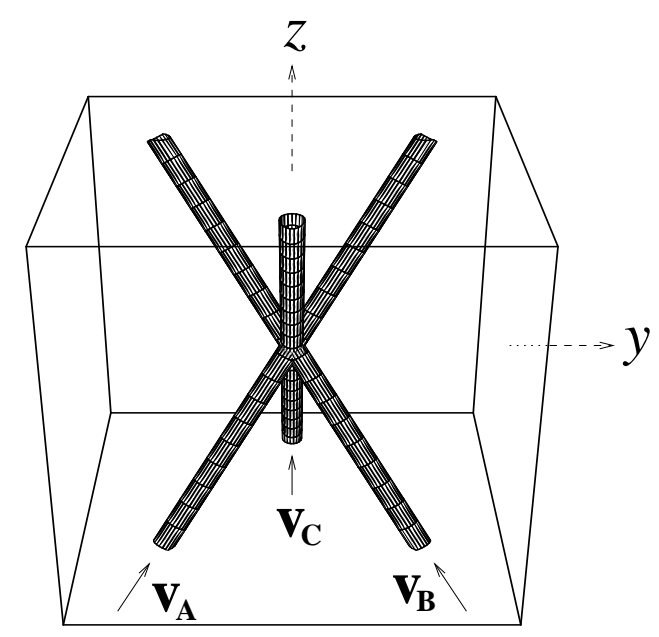

FIG. 1. A schematic description of the physical situation. We note that a particular geometry of the scheme and the type of the photonic crystal are not essential. The important feature of the crystal geometry is that it has straight "tunnels" so that atoms can traverse across the crystal. For concreteness, we choose the crystal with the geometry proposed by Yablonovitch et al. (references in Section III). We display only three of many cylindrical holes in the crystal. The cylindrical holes are drilled at the angle $\Theta=35.26^{\circ}$ with the vertical axis. We assume (except Section III) that there is a defect of the crystal periodicity near the region where the holes are crossing. This defect is responsible for a single defect mode localized in the center of the crystal. The frequency of the defect mode lies inside a wide photonic bandgap of the crystal. Linear dimensions of the defect-mode region are comparable with the lattice constant of the structure. The atoms $A, B$ (and $C$, if needed) are injected into the three holes at the bottom side of the crystal at (approximately) the same time and with suitably adjusted velocities. The atoms are assumed to be two-level systems with their transition frequencies equal to the defect-mode frequency. We assume that transitions including other atomic levels can be neglected. The atom $A$ is injected in its upper level $\left|e_{A}\right\rangle$ and the atom $B$ and $C$, in their lower levels $\left|g_{B}\right\rangle$ and $\left|g_{C}\right\rangle$. The initial state of the defect mode is vacuum state. The states of the atoms are detected at the exit from the crystal. We consider the following numerical values for the setup. The crystal is a cube of the side $L \approx 20 \mathrm{~cm}$. The frequencies of the defect mode and the atomic transition are $\omega_{0} /(2 \pi)=\omega /(2 \pi)=21.50651 \mathrm{GHz}$, i.e. the same as transitions used in experiments with microwave cavities. This frequency lies inside the wide photonic bandgap if the crystal is made from dielectric with refractive index (at microwaves) 3.6 , volume filling fraction is $78 \%$ and the side of an elementary cube is $a \approx 16.3 \mathrm{~mm}$. We use this value of $a$ to calculate the parameter $k=\pi / a$ [see Eq.(12)]. 


$$
|\Psi(t)\rangle=a(t)\left|e_{A}, g_{B}, g_{C}, 0\right\rangle+b(t)\left|g_{A}, e_{B}, g_{C}, 0\right\rangle+c(t)\left|g_{A}, g_{B}, e_{C}, 0\right\rangle+\gamma(t)\left|g_{A}, g_{B}, g_{C}, 1\right\rangle
$$

where $t$ is the time at which we detect the internal states of the atoms at the exit of the crystal. The final values of the amplitudes $a, b, c$ and $\gamma$ depend on a particular setup of the experiment including the coupling parameters and velocities of the atoms. For completeness of the description we specify trajectories $\mathbf{r}_{j}(t)$ of the three atoms which can move along the axes of the three void regions

$$
\mathbf{r}_{j}(t)=\mathbf{r}_{j}(0)+\mathbf{v}_{j} t ; \quad j=A, B, C
$$

with the vectors $\mathbf{r}_{j}(0)$ and $\mathbf{v}_{j}$ specified by their components as

$$
\begin{aligned}
\mathbf{r}_{A}(0) & =\frac{L}{4}\{\tan \Theta,-\sqrt{3} \tan \Theta,-2\}, \\
\mathbf{v}_{A} & =\frac{v_{A}}{2}\{-\sin \Theta, \sqrt{3} \sin \Theta, 2 \cos \Theta\},
\end{aligned}
$$

for the atom $A$. While for the other two atoms ( $B$ and $C)$ we have

$$
\begin{aligned}
\mathbf{r}_{B}(0) & =\frac{L}{4}\{\tan \Theta, \sqrt{3} \tan \Theta,-2\} \\
\mathbf{v}_{B} & =\frac{v_{B}}{2}\{-\sin \Theta,-\sqrt{3} \sin \Theta, 2 \cos \Theta\} ;
\end{aligned}
$$

and

$$
\begin{aligned}
\mathbf{r}_{C}(0) & =\frac{L}{2}\{-\tan \Theta, 0,-1\} ; \\
\mathbf{v}_{C} & =v_{C}\{\sin \Theta, 0, \cos \Theta\} .
\end{aligned}
$$

Here we assume the origin of the coordinates in the center of the cube crystal with the side of the length $L ; \Theta$ is the angle between the axes of the cylinders and the $z$ direction.

\section{ENTANGLEMENT VIA RESONANT DIPOLE-DIPOLE INTERACTION}

In this Section we consider just two identical atoms ( $A$ and $B$ ) which move in the crystal as it is described above. Here we assume that there is no defect mode in the crystal. The atoms move inside the crystal with constant velocities. The recoil effect due to interaction with electromagnetic field is neglected because the atoms are relatively heavy particles. The interaction between the atoms and the electromagnetic field modes inside the crystal is described by the Hamiltonian in the electricdipole approximation

$$
\begin{aligned}
H & =\hbar \omega \sum_{j=A, B} \sigma_{z}^{j}+\hbar \sum_{\lambda} \omega_{\lambda} a_{\lambda}^{\dagger} a_{\lambda} \\
& -\frac{1}{\epsilon_{0}} \mu(A) \cdot \mathbf{D}\left(\mathbf{r}_{A}\right)-\frac{1}{\epsilon_{0}} \mu(B) \cdot \mathbf{D}\left(\mathbf{r}_{B}\right),
\end{aligned}
$$

where $a_{\lambda}$ and $a_{\lambda}^{\dagger}$ are the annihilation and creation operators of the field mode labeled by $\lambda, \mathbf{D}(\mathbf{r})$ is the transverse displacement-field operator and $\mu(A)$ and $\mu(B)$ are the atomic dipole operators. When the atomic transition frequencies are far from abrupt changes in the density of modes the Hamiltonian (7) can be approximated as (for more details see [18])

$$
H_{\mathrm{eff}}=\hbar \omega \sum_{j=A, B} \sigma_{z}^{j}+\hbar\left(J_{A B} \sigma_{+}^{A} \sigma_{-}^{B}+J_{B A} \sigma_{-}^{A} \sigma_{+}^{B}\right),
$$

where $\sigma_{ \pm}^{x}$ are raising and lowering operators of the atoms $(x=A, B)$ and $J_{A B}$ is a matrix element for the effective description of the RDDI [17]. For qualitative estimations, we will use $J_{A B}$ evaluated under the assumption that density of electromagnetic modes is that of a free space. In this case we find (for more details see [20])

$$
\hbar J_{A B}=\mu_{i}^{g e}(A) \mu_{j}^{e g}(B) \frac{1}{4 \pi \epsilon_{0} R^{3}}\left[\left(\delta_{i j}-3 \hat{R}_{i} \hat{R}_{j}\right)\left(\cos k_{A} R+k_{A} R \sin k_{A} R\right)-\left(\delta_{i j}-\hat{R}_{i} \hat{R}_{j}\right) k_{A}^{2} R^{2} \cos \left(k_{A} R\right)\right],
$$

where $R$ is the distance between the atoms, $k_{A} \equiv \omega / c$, $\mu^{e g}$ is the absolute value of the atomic dipole matrix element, and $\hat{R}_{i}$ are the components of the unit vector starting at the position of the atom $A$ and oriented towards the atom $B$. We assume summation over repeated indeces. We stress that the above expression for $J_{A B}$ is valid in a free space, but in the limit $R \ll \lambda$ it can also be applied for photonic crystals [17], i.e. it can be also used for an order-of-magnitude description of the RDDI effects in photonic crystals. These effects are most important in the regime $R \ll \lambda$ when the free-space expression is valid also in photonic crystal. We will apply the Hamil- tonian (8) with $J_{A B}$ given by Eq.(9) also for description of propagation of the atoms in the crystal in the case when $R \geq L$. Even though the expression for $J_{A B}$ given by Eq.(9) is not precise it provide us with a rather good picture of the RDDI effect. We note that in order to find more appropriate expression for $J_{A B}$ we would have to know the electromagnetic eigenmodes for the threedimensionally periodic structure and the corresponding derivation of $J_{A B}$ is very complicated.

In what follows we study the time evolution of the atoms initially prepared in the state $|\Psi(0)\rangle=\left|e_{A}, g_{B}\right\rangle$ which is governed by the effective Hamiltonian (8) with 
time-dependent $J_{A B}$ (which is due to the fact that the atoms are moving through the crystal). We show that the RDDI can in principle be used for controlling the entanglement between atoms. We have solved the corresponding Schrödinger equation numerically. We have used parameters typical for Rydberg atoms and currently existing photonic crystals. In Fig.2 we plot results for the time-dependent atomic populations. We have chosen the atomic trajectories similarly as it is specified in the previous section but we added a small value $(0.05-0.3 \mathrm{~mm})$ to the initial $x_{A}(0)$ coordinate so that the trajectory of atom $\mathrm{A}$ is parallel but not identical with the axis of the cylinder. This prevents the collision of the atoms. The velocities of both atoms are $200 \mathrm{~m} \mathrm{~s}^{-1}$.

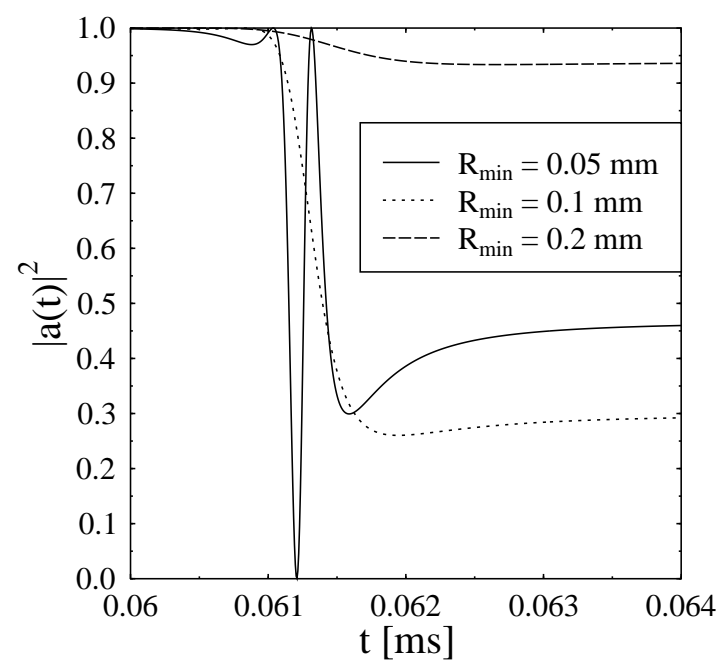

FIG. 2. The time evolution of the population of the upper level of the atom $A$ if the atoms interact according to effective Hamiltonian (8). The atoms move along the trajectories specified in Section [1]. Three curves correspond to three different values of $x_{A}(0)$ specified by the minimal distance $R_{\min }$ of the atoms during the passage. Both atomic dipoles are oriented in the $x$-direction. We evaluate the interaction only during the time interval when the atoms move near the center of the crystal in the cubic region of the side $2 \mathrm{~cm}$. The atomic velocities are $v_{A}=v_{B}=200 \mathrm{~m} \mathrm{~s}^{-1}$ and $\mu^{e g} / e \approx 6.7210^{-7} \mathrm{~m}$, where $e$ is the proton charge.

Taking into account that the physical conditions are chosen such that the electromagnetic field is adiabatically eliminated from the interaction [see the effective Hamiltonian (8)] the two atoms due to the unitarity of the evolution remain in a pure state $|\Psi(t)\rangle_{A B}=$ $a(t)\left|e_{A}, g_{B}\right\rangle+b(t)\left|g_{A}, e_{B}\right\rangle$ with the amplitudes $a(t)$ and $b(t)$ which depend on the RDDI. From here it follows that due to the RDDI the two atoms become entangled. The degree of entanglement in the present case can be quantified with the help of the von Neumann entropy $S=-\operatorname{Tr}[\hat{\rho} \ln \hat{\rho}]$ of each individual atom for which we have $S=-|a(t)|^{2} \ln |a(t)|^{2}-|b(t)|^{2} \ln |b(t)|^{2}$ where $|a(t)|^{2}=1-|b(t)|^{2}$. In other words the degree of the entanglement depends on the population of internal levels of the atoms and highest degree of entanglement is attained for $|a(t)|^{2}=|b(t)|^{2}=1 / 2$.

As seen from Fig. 2 the population of the excited state of the atom $A$ depends on the minimal distance $R_{\text {min }}$ between the atoms during the passage through the crystal. From our numerical investigation it follows that the atoms are most entangled for $R_{\min } \simeq 0.05 \mathrm{~mm}$. However we note that with present techniques the controle over the position of atoms in the configuration considered here is about $\pm 1 \mathrm{~mm}[6]$. Consequently, the RDDI is not very suitable for a coherent controle of entanglement between atoms in photonic crystals. In the following Section we consider entanglement via a defect mode when the currently available precision control is sufficient.

\section{ENTANGLEMENT VIA A DEFECT MODE}

Let us consider the interaction of the atoms with a single defect-field mode in the dipole and the rotating-wave approximations. We assume that the distance between the atoms is always sufficiently large so that they do not interact via RDDI. The corresponding Hamiltonian can be written as

$$
\begin{aligned}
H & =\hbar \omega \sum_{j=A, B, C} \sigma_{z}^{j}+\hbar \omega_{0} a^{\dagger} a \\
& +\hbar \sum_{j=A, B, C}\left[G\left(\mathbf{r}_{j}\right) \sigma_{+}^{j} a+G^{*}\left(\mathbf{r}_{j}\right) \sigma_{-}^{j} a\right],
\end{aligned}
$$

where $\omega_{0}$ is the mode frequency (which we assume to be equal to the atomic transition frequency $\omega), \sigma_{ \pm}^{j}$ are atomic raising and lowering operators and $\mathbf{r}_{A}$ and $\mathbf{r}_{B}$ are the positions of the atoms. The position dependence of the coupling parameters $G\left(\mathbf{r}_{j}\right)$ can be expressed as

$$
G\left(\mathbf{r}_{j}\right)=G_{0} \epsilon \cdot \mathcal{D}_{j} f\left(\mathbf{r}_{j}\right),
$$

where $f(\mathbf{r})$ is the field-mode amplitude at the position $\mathbf{r}, \epsilon$ is the electric-field polarization direction of the defect mode and $\mathcal{D}_{j}$ is a unit vector in the direction of the atomic dipole matrix element of the atom $j$. It is known that the spatial dependence of a defect-mode amplitude is a function which oscillates and decays exponentially [12]. A particular profile of the spatial dependence of the defect mode can be adjusted via a properly generated defect of the periodicity. A rigorous calculation of the electromagnetic field in the presence of a defect in a 3D photonic crystal can be a difficult task. In this paper we use a model profile of the spatial dependence of the electric field. Similar profiles have already been created in existing photonic crystals [11 14]. We note that for the purpose of the proposed experiment a complete information about the mode shape is not needed. The results of the experiment depend only on the shape along the trajectories of the atoms. In what follows we use the profile 


$$
f(\mathbf{r})=\exp \left[-\frac{\left|\mathbf{r}-\mathbf{R}_{0}\right|}{R_{\text {def }}}\right] \sin (\mathbf{k} \cdot \mathbf{r}+\Phi),
$$

where $\mathbf{R}_{\mathbf{0}}$ is the position around which the mode is localized, $R_{\text {def }}$ is a parameter (defect-mode radius) describing the rate of the exponential decay of the mode envelope, $\Phi$ is a phase factor and $\mathbf{k}$ is the parameter describing spatial oscillations of the field mode. We chose its magnitude to be $k=\pi / a$ where $a$ is the value of the side of an elementary cubic cell in the photonic crystal. We consider values of the constant $R_{\text {def }}$ comparable with $a$. We estimate the value of the coupling constant $G_{0}$ from microcavity experiments 16

$$
G_{0}=\sqrt{\frac{V_{\text {cav }}}{V_{\text {eff }}}} \Omega,
$$

where $V_{\text {cav }}$ is the modal volume of the microcavity mode, $V_{\text {eff }}$ is the effective modal volume of the defect mode and $\Omega$ is the vacuum Rabi frequency in the microwave experiment. The numerical values are [16]: $V_{\text {cav }}=11.5 \mathrm{~cm}^{3}$ and $\Omega=43 \mathrm{kHz}$. When we consider the transitions between levels $63 P_{3 / 2}$ and $61 D_{3 / 2}$ of Rubidium atoms, then the atomic transition frequency is $\omega /(2 \pi)=21506.51$ $\mathrm{MHz}$. Finally, the effective modal volume can be approximated as

$$
V_{\mathrm{eff}}=\frac{4}{3} \pi\left(2 R_{\mathrm{def}}\right)^{3}
$$

Because the atoms are moving the coupling parameters depend on time [in what follows we will use the notation $\left.G_{j}(t)\right]$ We consider positions of the atoms given by Eqs.(4) and (5). In some cases we add a small value to $x_{A}(0)$ given by (4) to prevent the atoms to collide in the center of the crystal. Details of the geometry of the proposed experiment are given in Section II and in Fig. 1.

Once we have specified all model parameters we can solve the Schrödinger equation for the system which is supposed to be initially prepared in the state $|\Psi(0)\rangle=$ $\left|e_{A}, g_{B}, g_{C}, 0\right\rangle$. Due to the fact that the number of excitations is an integral of motion in the present case the state vector at time $t>0$ has the form (2) and the corresponding Schrödinger equation can be rewritten into a set of a system of linear differential equations. These equations can be solved analytically for time-independent coupling constants $G_{j}(t)$ which is not our case. Therefore we have to integrate the equations numerically.

\section{A. One atom}

We start our discussion with a problem when just a single atom (let say the atom $A$ ) passing through the crystal is considered. We assume that the atom is on resonance with the defect mode (i.e., $\omega=\omega_{0}$ ).
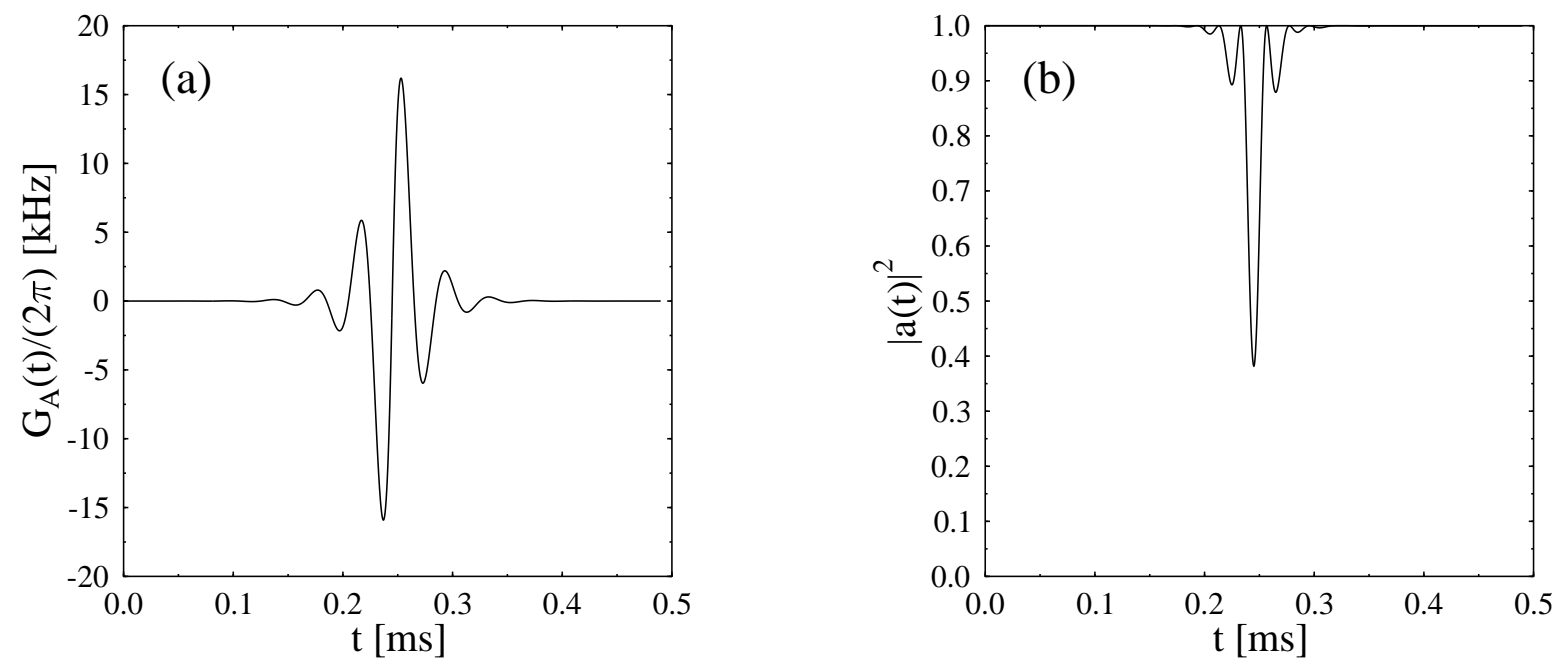

FIG. 3. (a) The time dependence of the coupling $G_{A}(t)$ between the defect mode and the single atom $A$ when it moves along the axis of the cylinder with the velocity $v_{A}=500 \mathrm{~ms}^{-1}$. The mode position and the geometry is given by the parameters $\mathbf{R}_{\mathbf{0}}=\mathbf{0}, \Phi=0 \mathrm{rad}$ and $R_{\text {def }}=10 \mathrm{~mm}$. The parameter $\mathbf{k}=(0,0, \pi / a)$ where $a \approx 16.3 \mathrm{~mm}$. The atomic dipole is oriented in the $x$ direction (same as the field polarization). The integral (17) in this case is equal to zero. Consequently, the atom at the exit of the crystal is again in its initial state. We plot the time evolution of the population of the exited level of the atom in Fig.b.

This corresponds to the Jaynes-Cummings model 21 with a time-dependent coupling constant. The general solution of this model for real coupling parameter was found Sherman et al. 22. With the initial condition $|\Psi(0)\rangle=\left|e_{A}, 0\right\rangle$ the solution can be expressed as 


$$
\begin{aligned}
|\Psi(t)\rangle & =\cos \left[\int_{0}^{t} G_{A}\left(t^{\prime}\right) d t^{\prime}\right]\left|e_{A}, 0\right\rangle \\
& -i \sin \left[\int_{0}^{t} G_{A}\left(t^{\prime}\right) d t^{\prime}\right]\left|g_{A}, 1\right\rangle .
\end{aligned}
$$

This implies for the atomic excitation

$$
P_{\mathrm{e}}^{(A)}(t)=\cos ^{2}\left[\int_{0}^{t} G_{A}\left(t^{\prime}\right) d t^{\prime}\right] .
$$

In the case of the defect mode with linear dimensions much smaller than the side of the crystal we can use the approximation

$$
\int_{0}^{t} G_{A}\left(t^{\prime}\right) d t^{\prime} \approx \int_{-\infty}^{\infty} G_{A}\left(t^{\prime}\right) d t^{\prime} .
$$

We note that this integral for a given choice of the profile function [see Eq.(12)] with the phase of the field mode $\Phi=0$ equals to zero. This means that the atom exits the crystal in the same state as it entered it. Obviously the defect mode also remains in its initial (vacuum) state. In Fig.3a we plot the time dependence of the coupling constant between the atom $A$ and the defect mode. While in Fig. $3 \mathrm{~b}$ we present the time dependence of the corresponding excited-state probability. It is assumed that the defect is located at the center $\mathbf{R}_{0}=\mathbf{0}$ of the crystal. The other parameters are chosen such that $\Phi=0 \mathrm{rad}$, $\mathbf{k}=(0,0, k), \mathcal{D}_{A}=\epsilon=(1,0,0)$ [see Eqs. (11),(12)]. We assume that the atom moves along the axis of the cylindrical cavity. The velocity of the atom is chosen to be $v_{A}=500 \mathrm{~ms}^{-1}$. From Fig. 3a we clearly see that the atom on its way through the crystal interacts with the defect mode just around the center of the crystal. The other important feature is seen from Fig. 3 b, i.e. The atom is transiently entangled with the defect mode in the center of the crystal. Nevertheless it leaves the crystal in a pure (unentangled) state. This effect of "spontaneous" disentanglement of the atom from the defect mode is very important when we consider creation of pure entangled state of two atoms.

\section{B. Two atoms}

Let us consider a situation when two atoms interact with the same defect mode as in the previous case. The atoms have their dipoles oriented along the direction $\epsilon$ of the electric-field polarization. The velocity of the atom $A$ is $500 \mathrm{~ms}^{-1}$. The time evolution of the corresponding atomic populations for various velocities of the atom $B$ are plotted in Fig. 4 .

Firstly we consider both atoms to have the same velocity (see Fig. (Aa). In this case we assume that the atom $A$ is displaced from the axis of the cylindrical hole through which it flies [i.e. we add $0.3 \mathrm{~mm}$ to $x_{A}(0)$ given by (4)] to avoid the influence of the RDDI between the atoms and their collision. We see that the atoms strongly interact with the field in the region of the defect. However, after the interaction the initial state of the system is approximately restored (see the "stationary" values of the probability amplitudes $a(\tau), b(\tau)$ and $\gamma(\tau)$ which are displayed in the figures). It is interesting to compare Fig. $3 \mathrm{~b}$ with Fig. 伟 to see how the time evolution of the population of the atom $A$ is modified by the presence of the additional atom $B$. We see that for the given set of parameters the presence of the atom $B$ does not influence the dynamics of the atom $A$ significantly.

Now we will study how the level population depends on the velocity of the atom $B$. From Fig. A we see that for properly chosen velocity the interaction between the atoms mediated by the defect field can be pronounced.

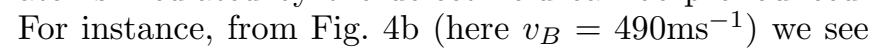
that not only the excitation of the atom $B$ can be higher than the population of the atom $A$, but also the defect mode becomes partially excited and entangled with the atomic system.

When the atom $B$ has the velocity $v_{B}=515 \mathrm{~ms}^{-1}$ (see Fig. $4 c$ c) then the defect mode in the stationary limit is in the vacuum state $[\gamma(\tau) \simeq-0.0616 i]$ and is (with high precision) completely disentangled from the atomic system. It is interesting to note that in this particular situation the defect mode mediates transfer of most of the excitation from the atom $A$ to the atom $B$.

Let us assume now the velocity of the atom $B$ to be $v_{B}=532.8 \mathrm{~ms}^{-1}$ (see Fig. 19d). In this case the defect mode in the stationary limit is again in the vacuum state and is completely disentangled from the atomic system. Moreover the amplitudes $a(\tau)$ and $b(\tau)$ are in this case almost equal, which means that the atoms at the exit from the crystal are in the state $|\Psi\rangle=\left(\left|e_{A}, g_{B}\right\rangle+\left|g_{A}, e_{B}\right\rangle\right) / \sqrt{2}$, i.e. they are prepared in a pure maximally entangled state.

In the cases presented in Fig. A the phase factor $\Phi$ of the defect mode is set to zero so that the integrals of the coupling constants $G_{A}(t)$ and $G_{B}(t)$ over the trajectories of the atoms are equal to zero. The defect-mode radius $R_{\text {def }}=10 \mathrm{~mm}$. We have also studied the dynamics for other values of $\Phi$, when the integrals of the coupling constants differ from zeros. In this case the disentanglement of the defect mode and the atoms is not so well pronounced, i.e. the defect mode becomes excited. We have also found a general feature: If the integrals of coupling constants are zeros and the coupling constants are small enough then the defect mode after the interaction is left in the vacuum state. However, if we increase the couplings (by decreasing the mode volume $V_{\text {eff }}$ ) the defect mode can be left in an excited state [i.e. $\gamma(\tau) \neq 0$; see the expression for the state vector (2)]. Consequently, the atoms are left in a mixture state.

We have also analyzed the situation when the defect mode is not located directly at the center of the crystal. Moreover we have assumed that $\Phi \neq 0$. It can be shown that even in this case it is possible to find a value $v_{B}$ at which the atoms exit the crystal in a nearly pure maximally entangled state. 

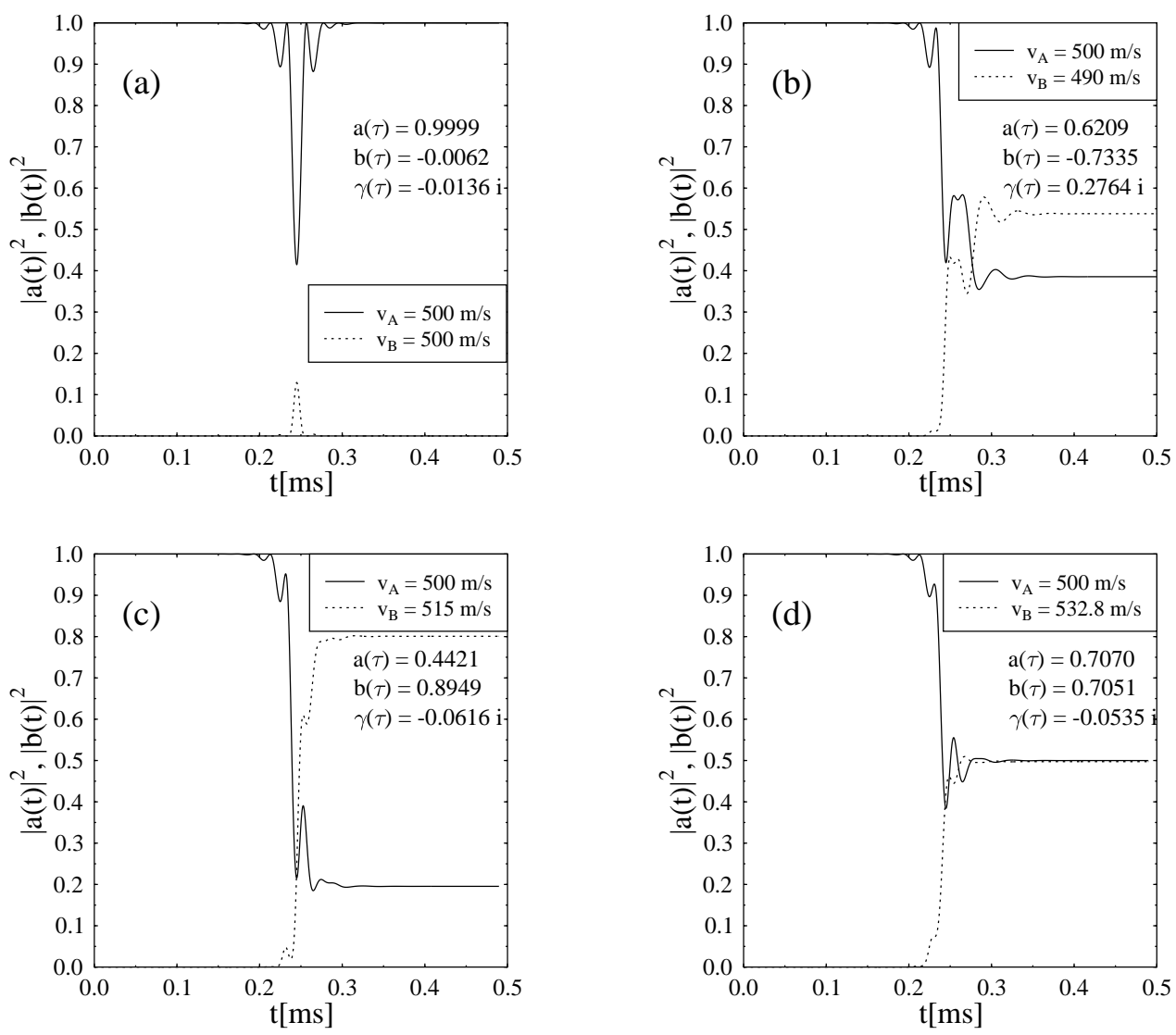

FIG. 4. The time evolution of the populations of excited levels of the atoms $A$ (solid line) and $B$ (dashed line). The atom $A$ enters the crystal in the excite state, while the atom $B$ is initially in the ground state. The atoms are injected into the crystal at the same time (see the setup presented by Fig.11) with the velocity of the atom $A$ being $v_{A}=500 \mathrm{~ms}{ }^{-1}$ and $R_{\text {def }}=10 \mathrm{~mm}$. The four plots corresponds to four different velocities of the atom $B$ (their numerical values are shown in figures). In the case when the two atoms are assumed to have equal velocities (Fig a) we add a small value (0.3 mm) to the initial position $x_{A}(0)$ [see the expression (4)] to avoid the collision of the atoms in the center of the crystal. The other parameters are chosen same as in Fig. 3. We write final values of the probability amplitudes into figures. From Fig. (d) we see that for properly chosen velocities of the atoms and times large enough (i.e. the atoms have already left the crystal) the defect mode is approximately in the vacuum state (it is disentangled from the atoms) and the atoms are prepared in a pure superposition state $|\Psi\rangle \simeq\left(\left|e_{A}, g_{B}\right\rangle+\left|g_{A}, e_{B}\right\rangle\right) / \sqrt{2}$.

\section{Three atoms}

Let us consider the same setup as in our previous discussion except we assume now three atoms flying through the crystal (see Fig. 11). These three two-level Rydberg atoms $(A, B$ and $C)$ are injected into the holes at the bottom side of the crystal simultaneously. The atom $A$ is initially in its upper level $\left|e_{A}\right\rangle$ while atoms $B$ and $C$ are initially in their lower states $\left|g_{B}\right\rangle$ and $\left|g_{C}\right\rangle$. The single defect mode is initially prepared in its vacuum state $|0\rangle$. The atoms move along the axes of the holes and interact with the defect mode in the central region of the crystal. The electric-field amplitude of the mode is given by Eq. (12). We consider slightly asymmetric position of the defect mode in the crystal (the reason is explained below). In Fig. [] we present plots of the final atomic populations versus velocities $v_{B}$ and $v_{C}$ while $v_{A}$ is fixed at the value $500 \mathrm{~ms}^{-1}$. These plots show that adjusting atomic velocities we can obtain required probabilities such that in the final state (2) the probability amplitude $\gamma(\tau)$ is equal to zero, which means that the defect mode is decoupled from the atomic system. The atoms are then in a pure superposition state. In particular, if we select velocities $v_{B}=536.4 \mathrm{~ms}^{-1}$ and $v_{C}=527.4 \mathrm{~ms}^{-1}$, we obtain a final state with equal probabilities $|a(\tau)|^{2}=|b(\tau)|^{2}=|c(\tau)|^{2} \approx 0.33$ (see Fig.6). Square of $|\gamma(\tau)|$ gives the probability of the photon in the final state approximately equal to 0.02 . It means that the atomic subsystem is in a good approximation decoupled from the field subsystem. 

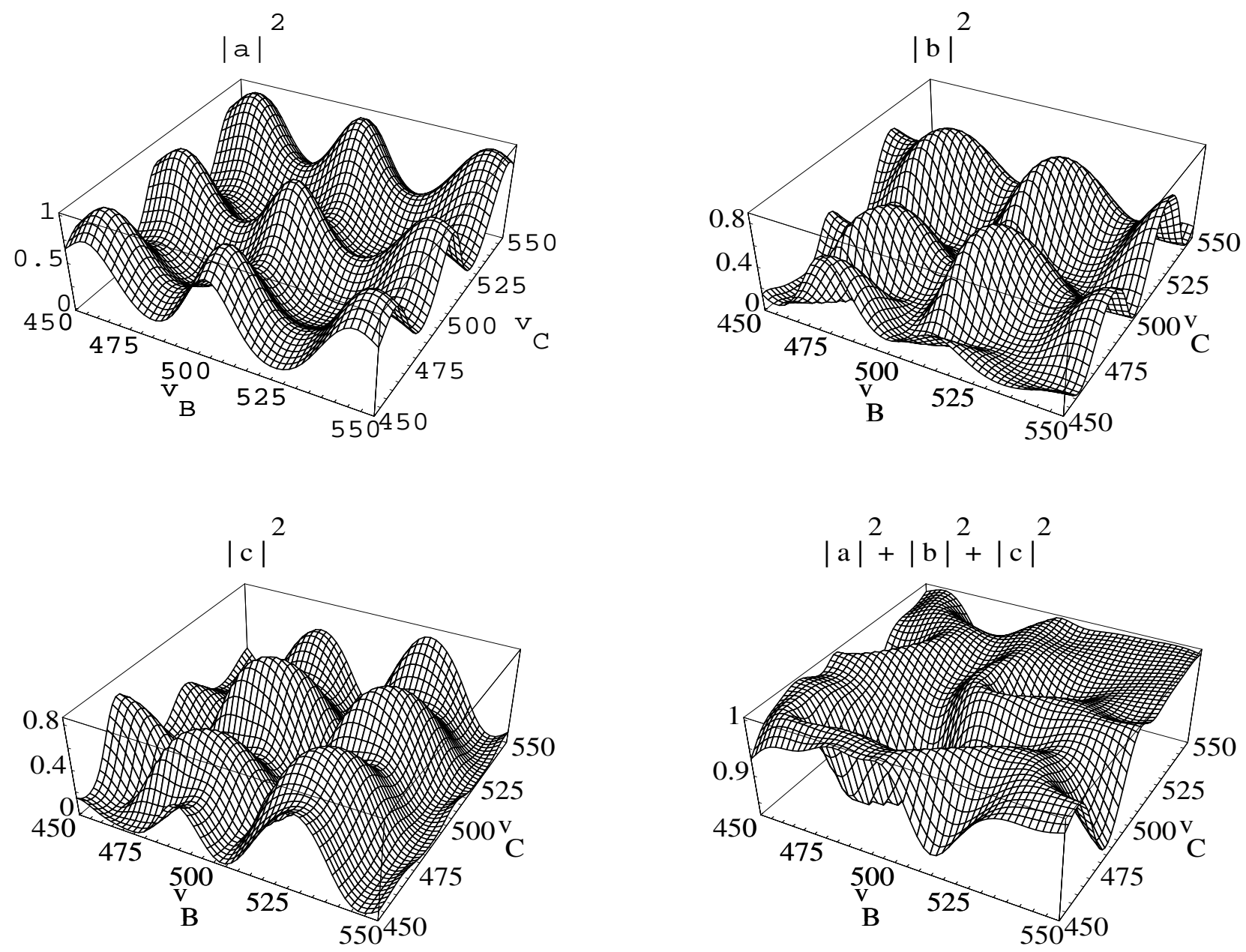

FIG. 5. Final atomic populations of the three atoms which interact with the defect mode in the crystal versus velocities $v_{B}$, $v_{C}$ at fixed value $v_{A}=500 \mathrm{~ms}^{-1}$. The defect region is positioned unsymmetrically: $\mathbf{R}_{0}=(1,-3,2) \mathrm{mm}$. We chose the phase $\Phi=0$ and the other parameters $\left(a\right.$ and $\left.R_{\text {def }}\right)$ same as in Fig. 3 .

We have chosen an asymmetric position of the defect mode with respect to the center of the crystal because for the symmetric position we were able to obtain the "symmetric" result $|a(\tau)|^{2}=|b(\tau)|^{2}=|c(\tau)|^{2} \approx 0.33$ only when two of the velocities are equal. In this case we face the problem of the collision of the atoms. We expect that a better choice of the defect geometry might produce a final state more disentangled from the field as is the case presented in Fig.6.

We see from Figs. 5 that variations of the final atomic populations are rather robust with respect to changes in velocities, i.e. uncontrolled velocity fluctuations (which in experiments can be reduced up to $0.4 \mathrm{~ms}^{-1}$ [6]) do not deteriorate the predicted entanglement.

\section{CONCLUSIONS}

In this paper we have shown that atoms can be entangled in photonic crystals via dipole interaction mediated by off-resonant modes or via an interaction with a single defect mode. In the first mechanism (RDDI) the atoms can coherently exchange excitation while only a very small part of this energy is radiated into the field. However, this interaction might not be easy to control in an experiment because it requires a high precision position control of the position of the atoms. The second mechanism (via a single resonant defect mode) is experimentally more promising because it can be realized with currently available microwave photonic crystals and with highly excited Rydberg atoms.

We have shown that atoms can be prepared in pure entangled states and that the probability amplitudes of the generated superposition states of the atoms can be coherently controlled by varying the velocities of the atoms or by varying the orientations of the atomic dipole matrix elements.

In our scheme of entanglement via defect modes in photonic crystals the distance between the entangled atoms at the exit from media depends on the size of the media, the angle between the atomic trajectories, the atomic velocities and the life of the atoms. For the parameters used in this paper the distance between the entangled atoms is of the order of tens of centimeters. 
Finally, we think that investigation of dynamics of Rydberg atoms in photonic crystals is an interesting complement to current experimental cavity quantum electrodynamics.

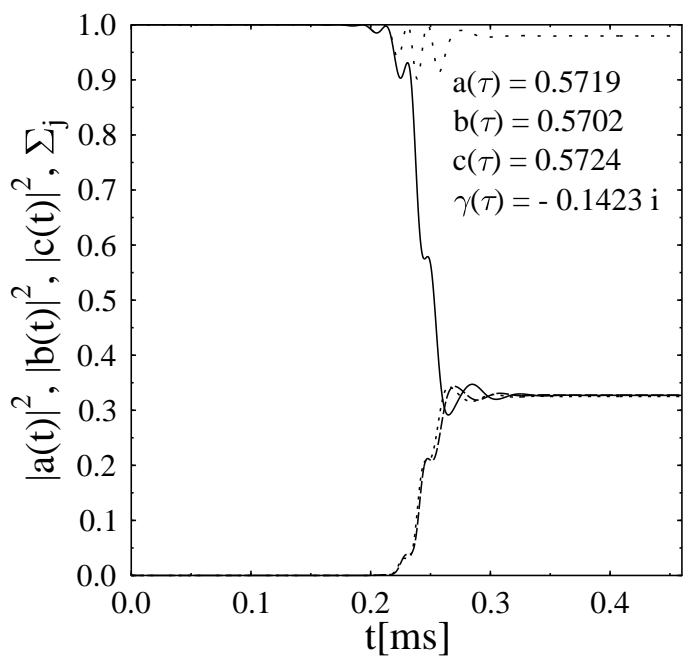

FIG. 6. The time evolution of the atomic populations $|a(t)|^{2}$ (solid line), $|b(t)|^{2}$ (long dashed line), and $|c(t)|^{2}$ (short dashed line), when the three atoms are injected into the photonic crystal simultaneously with the velocities $v_{A}=500 \mathrm{~ms}^{-1}, v_{B}=536.4 \mathrm{~ms}^{-1}$ and $v_{C}=527.4 \mathrm{~ms}^{-1}$. All other parameters are the same as in Fig.5. We see that the three atoms exit the crystal in a state nearly disentangled from the field state - we plot the sum $|a(t)|^{2}+|b(t)|^{2}+|c(t)|^{2}$ (dotted line) which is close to unity.

[1] A. Einstein, B. Podolsky, N. Rosen, Phys. Rev. 47, 777 (1935).

[2] J.S. Bell, Physics 1, 195 (1964); Speakable and Un- speakable in Quantum Mechanics (Cambridge University Press, Cambridge, 1987).

[3] A. Aspect, P. Grangier, and G. Roger, Phys. Rev. Lett. 49, 1804 (1982).

[4] B.J. Oliver, C.R. Stroud, J. Opt. Soc. Am. B 4, 1426 (1987).

[5] S.J.D. Phoenix, S.M. Barnett, J. Mod. Opt. 40, 979 (1993); I.K. Kudryatsev, P.L. Knight, J. Mod. Opt. 40, 1673 (1993); J.I. Cirac, P. Zoller, Phys. Rev. A 50, R2799 (1994); M. Freyberger, P.K. Aravind, M.A. Horne, A. Shimony, Phys. Rev. A 53, 1232 (1996).

[6] E. Hagley, X. Maître, G. Nogues, C. Wunderlich, M. Brune, J.M. Raimond, S. Haroche, Phys. Rev. Lett. 79, 1 (1997).

[7] Q.A. Turchette, C.S. Wood, B.E. King, C.J. Myatt, W.M. Itano, C. Monroe, and D.J. Wineland, Phys. Rev. Lett. 81, 1525 (1998).

[8] E. Yablonovitch, Phys. Rev. Lett. 58, 2059 (1987).

[9] S. John, Phys. Rev. Lett. 58, 2486 (1987).

[10] E. Yablonovitch, T.J. Gmitter, K.M. Leung, Phys. Rev. Lett. 67, 2295 (1991).

[11] E. Yablonovitch, T.J. Gmitter, R.D. Meade, A.M. Rappe, K.D. Brommer, J.D. Joannopoulos, Phys. Rev. Lett. 67, 3380 (1991).

[12] S.L. McCall, P.M. Platzmann, R. Dalichaouch, D. Smith, S. Schultz, Phys. Rev. Lett. 67, 2017 (1991).

[13] J.N. Winn, R.D. Meade, J.D. Joannopoulos, J. Mod. Opt. 41, 257 (1994).

[14] D.R. Smith, S. Schultz, S.L. McCall, P.M. Platzmann, J. Mod. Opt. 41, 395 (1994).

[15] E. Yablonovitch, J. Mod. Opt. 41, 171 (1994).

[16] D. Meschede, H. Walther, G. Müller, Phys. Rev. Lett. 54, 551 (1985).

[17] S. John, J. Wang, Phys. Rev. B 43, 12772 (1991).

[18] S. John, Tran Quang, Phys. Rev. A 52, 4083 (1995).

[19] G. Kurizki, Phys. Rev. A 42, 2915 (1990).

[20] D. P. Craig, T. Thirunamachandran, Molecular Quantum Electrodynamics (Academic, London, 1984).

[21] E.T. Jaynes, F. W. Cummings, Proc. IEEE 51 (1963) 89; for a review see B.W. Shore, P.L. Knight, J. Mod. Opt. 40 (1993) 1195.

[22] B. Sherman, G. Kurizki, A. Kadyshevitch, Phys. Rev. Lett. 69, 1927 (1992). 